\title{
La productividad científica sobre MOOC: aproximación bibliométrica 2012-2016 a través de SCOPUS
}

\section{Scientific productivity on MOOCs: 2012-2016 bibliometric approach through Scopus}

\author{
Santiago Mengual-Andrés \\ Universidad de Valencia (España) \\ Esteban Vázquez-Cano \\ Universidad Nacional de Educación a Distancia, UNED (España) \\ Eloy López Meneses \\ Universidad Pablo de Olavide (España)
}

\section{Resumen}

Los Massive Online Open Courses (MOOC) han supuesto un hito en la educación online en los últimos cinco años. La proliferación de cursos y plataformas para su difusión han hecho que el fenómeno no solo sea un punto de reflexión educativa, social y política, sino que también pase a la esfera académica como objeto de investigación. El presente estudio analiza la productividad científica del fenómeno MOOC a partir del análisis de 752 publicaciones indexadas en la base de datos SCOPUS en el periodo 2012-2016. Los datos extraídos permiten realizar una radiografía global del estado del fenómeno, la evolución que ha sufrido en los últimos años, el tipo de publicaciones que sustentan el fenómeno, los principales países y fuentes productores de información sobre MOOC, las principales filiaciones implicadas, los autores y trabajos más citados, así como un análisis de co-citación que permite vislumbrar los trabajos más destacados sobre el fenómeno. Por lo tanto, el presente estudio ofrece un marco complementario a diversos estudios existentes de carácter nacional e internacional que intentan ofrecer y explicar la configuración del mapa de la ciencia alrededor del fenómeno MOOC.

Palabras clave: MOOC; bibliometría; SCOPUS; productividad científica; educación a distancia.

\begin{abstract}
Massive Open Online Courses (MOOC) could be considered as a milestone on online education over the past five years. The proliferation of courses and platforms have made this phenomenon not only a matter of educational, social and political reflection, but also a scientific research topic. This study analyzes the scientific productivity of the MOOC phenomenon based on the analysis of 752 publications indexed in the SCOPUS database
\end{abstract}


during the period from 2012 to 2016. The data retrieved offers a general overview of the state of the art, the evolution throughout the recent years, the types of publications, the most representative countries and sources of information about MOOCs, the main institutional affiliations, the most cited authors and papers, as well as a co-citation analysis that presents the most outstanding works on MOOCs. Therefore, this study provides a complement to existing national and international studies that try to explain the configuration of a map of science around the MOOC phenomenon.

Keywords: MOOC; bibliometrics; SCOPUS; scientific production; distance education.

Los últimos años se están caracterizando por una apuesta formativa basada en actividades, cursos y propuestas que tienen como base procesos de enseñanza aprendizaje mediados por la tecnología (Castaño-Muñoz, Duart y Teresa, 2015; Estévez y García, 2015; Roig-Vila, Mondéjar y Lorenzo-Lledó, 2016; Colorado, Marín-Díaz y Zavala, 2016). En este contexto mediado por la tecnología, la irrupción de los cursos masivos, en línea y en abierto denominados con la sigla inglesa "MOOC" (Massive Open Online Course) en los diferentes ámbitos de la Educación y la Formación ha sido imparable (Cormier y Siemens, 2010; Siemens, 2012; Downes, 2012, 2013; Yuan y Powell, 2013; Dillenbourg et al., 2014; Daniel, Vázquez-Cano y Gisbert, 2015; Mengual-Andrés, Roig-Vila y Lloret Catalá, 2015). El impacto de la formación en línea se ha dejado sentir con especial fuerza en la Educación Superior pero también en los últimos años está teniendo especial relevancia en la enseñanza preuniversitaria y en los procesos formativos empresariales (Pappano, 2012; Anderson, 2013; Conole, 2013; Little, 2013; Vázquez-Cano, 2013; Vázquez-Cano, López-Meneses y Sarasola, 2013; Roig Vila, Mengual Andrés y Suárez Guerrero, 2014; Gisbert y Lázaro, 2015; Marín Díaz y Sampedro Requena, 2016).

El movimiento MOOC también ha desarrollado una numerosa bibliografía que abarca diferentes dimensiones de la formación masiva: modelos didácticos, tasas de abandono, certificación, acreditación, entre otras (Eaton, 2012; Zapata, 2013; Daniel, 2013; Guàrdia, Maina y Sangrà, 2013; Christensen et al., 2013; Sangrà, 2013; Hoxby, 2014; Radford et al., 2014; DeBoer et al., 2014; Ramírez-Fernández, 2015; Vázquez-Cano y Sevillano, 2015; Vázquez-Cano, López Meneses y Barroso Osuna, 2015).

En los últimos años se han publicado diferentes estudios bibliométricos que ayudan a entender la evolución del movimiento y que sirven a la comunidad científica para determinar que orientación está tomando esta tendencia formativa (Liyanagunawardena, Adams y Williams, 2013; López Meneses, Vázquez-Cano y Román, 2015; Aguaded, Vázquez-Cano y López Meneses, 2016). Este estudio complementa los ya publicados y aporta dos aspectos novedosos y diferenciadores con respecto a los estudios bibliométricos previos; por un lado, el análisis de un nuevo corpus de documentos: Conference Paper, Article, Book Chapter, Review Article in Press, Editorial Letter, Note, Short Survey y Book. Por otro lado, se ha empleado 
una técnica de análisis más precisa mediante el empleo del programa Bibexcel para la normalización de los datos y VosViewer para la visualización y representación de los nodos de datos.

El movimiento MOOC, al igual que cualquier ciencia o campo de estudio, requiere de procesos de recuperación, evaluación y análisis que brinden la posibilidad de visualizar y representar de forma exhaustiva, consistente, relevante y precisa el resultado de su labor y que asegure la legitimidad y originalidad del conocimiento científico producido. Por este motivo, el presente trabajo pretende ofrecer una panorámica global desde la cuantificación bibliométrica de la evolución de la productividad científica sobre el fenómeno MOOC a partir de la base de datos SCOPUS, en un periodo de cinco años comprendido entre 2012 y 2016. Las variables sometidas a análisis han sido: a) distribución general de la productividad MOOC por año y tipo de documento, b) distribución de la productividad por área temática y año, c) productividad por países, d) productividad por filiaciones, e) principales publicaciones periódicas, f) autores más citados, g) trabajos más citados, h) indicadores de co-citación.

\section{DISEÑO DE LA INVESTIGACIÓN}

La presente investigación se ha abordado a través de un análisis bibliométrico de la productividad científica respecto al movimiento MOOC, a través de estudio de trabajos del campo de las Ciencias de la Educación. Esta óptica de análisis no es exclusiva de este trabajo; su validez viene avalada por trabajos del campo de la educación que también se han abordado desde esta óptica (E.j: Fernández y Bueno, 1998; Ariza et al., 2011; Fernández, 2011; Gómez-García et al., 2012; Vázquez-Cano, López Meneses y Cobos Sánchiz, 2015; López Meneses, Vázquez-Cano y Sarasola, 2015; Zancanaro y Domingues, 2016). El análisis y tratamiento de datos se llevó a cabo a través de estadísticos descriptivos y medidas de tendencia central y dispersión (Peritz y Bar-Ilan, 2002; Davis y González, 2003), complementadas con técnicas centradas en el análisis de información en red (Knoke y Yang, 2008). Al igual que otros recientes trabajos (e.j: López-Meneses, Vázquez-Cano y Román, 2015) se seleccionó la base de datos internacional SCOPUS en base a: a) reconocimiento científico de la misma, b) amplia cobertura nacional e internacional de los trabajos. En dicho sentido, al ser menos restrictiva que WOS, permitió ofrecer una panorámica descriptiva con mayor cobertura de representación respecto al tema de estudio.

\section{PROCEDIMIENTO Y ANÁLISIS DE DATOS}

Durante el mes de abril de 2016 se llevó a cabo la búsqueda, recolección y cuantificación de las publicaciones científicas que respondían al fenómeno MOOC. La identificación de los trabajos se llevó a cabo a través de la base de datos SCOPUS, 
empleando operadores booleanos y opciones de búsqueda avanzada. Se decidió basar la búsqueda fundamentalmente en los campos TITLE-ABK_KEY (Título, Abstract y Palabras Clave), a través de la localización de términos distintos (MOOC, XMooc, cMOOC, OpenHPI, EdX, etc.), combinando los buscadores lógicos a través de diversas estrategias como:

(TITLE-ABS-KEY (mooc) OR TITLE-ABS-KEY (massive open online courses) OR TITLE-ABS-KEY (curso online masivo abierto) AND PUBYEAR > 2012 AND PUBYEAR < 2017 (TITLE (mooc) OR TITLE (massive open online courses) OR TITLE (curso online masivo abierto) AND PUBYEAR > 2011 ( KEY (mooc) AND NOT TITLE (mooc) AND PUBYEAR > 2011

A diferencia de otros estudios de reciente publicación (E.j; Zancanaro y Domingues, 2017; López-Meneses, Vázquez-Canoy Román, 2015; Aguaded, VázquezCano y López-Meneses, 2016), el presente trabajo analizó un corpus de trabajos más elevado y más entendido en el tiempo (2011-2016). Tras el procedimiento de búsqueda se obtuvo un corpus inicial de 1451 documentos que fue almacenado en una lista de la base de datos. A través de un análisis cualitativo se revisó el corpus inicial con objeto de filtrar los artículos no vinculados con la temática de estudio y seleccionar solo aquellos que podían responder al contenido de estudio en función del contenido del resumen. El resultado del análisis devolvió una muestra definitiva de 752 documentos de entre los años 2012 y 2016. Los documentos recuperados retornaron información suficiente para emprender el estudio bibliométrico en base a las tendencias actuales (Alcaide, Far e Iglesias, 2014; Zancanaro, Todesco y Ramos, 2015). La información recuperada aportó información sobre: a) fuente de publicación, b) tipo de documento, c) localización geográfica, d) afiliación institucional, e) citas recibidas, f) palabras clave, g) año de publicación, h) área de conocimiento e i) referencias citadas en los documentos. El corpus definitivo de referencias fue exportado mediante CSV, se empleó Bibexel para la normalización de los datos y VosViewer para la visualización y representación de los nodos de datos. Finalmente, los análisis descriptivos sobre indicadores bibliométricos de producción (productividad de los autores, tipo de documentos, años, citas, etc.) se llevó a cabo con el paquete estadístico SPSS 22 y fueron completados con un análisis de cocitación. 


\section{RESULTADOS Y DISCUSIÓN}

\section{Indicadores iniciales de producción}

\section{Distribución general de la productividad MOOC por año}

En un primer análisis, se puede observar como la productividad respecto a la investigación de los MOOC ha tenido un crecimiento exponencial en los últimos años. Los 752 trabajos indexados en SCOPUS marcan los años 2012 y 2013 como el inicio del proceso de publicación científica del fenómeno MOOC; con un $0,8 \%(n=6)$ y un $12.1 \%(n=91)$ respectivamente. Sin embargo, la mayor concentración hasta el momento ha sido descrita entre el año 2014, con un 33,1\% de las publicaciones hasta el momento $(n=249)$ y en particular en 2015 , con un $42,4 \%(n=319)$ de las publicaciones totales. En esencia, la tendencia del año 2016, con un 11.6\% ( $n=87)$ podría ser una evidencia de la desaceleración del fenómeno (era post-MOOC) u otros factores asociados como el retraso en la indexación de trabajos del último trimestre por parte de SCOPUS.

\section{Distribución general de la productividad por tipo de documento y año}

Como bien se justificó al inicio, SCOPUS ofrece una amplia cobertura que queda reflejada en su "Content Coverage List" (2016). En dicho sentido, los datos globales sobre producción científica alrededor de MOOC se han distribuido en mayor medida a través de dos grandes tipologías de documentos (tabla 1). El 41,3\% de la productividad $(\mathrm{n}=301)$ ha estado bajo el formato de artículo. En dicho cómputo incluimos también los artículos en prensa $(n=16)$ y los artículos de revisión $(n=21)$. Hemos excluido de este cómputo el 1.6\% $(n=12)$ de editoriales. Otro gran bloque de trabajos sobre MOOC estaría conformado por los "conference papers" que, con 358 documentos, confirman un total del $47,6 \%$ de los trabajos publicados.

Por lo que respecta a los libros por compilación y capítulos de libro indexados en SCOPUS encontramos que representan el 5,9\% $(n=44)$, siendo dicha publicación algo mayor que el resto de documentos que representan el $3,3 \%$ de la productividad (cartas al editor, notas y cuestionarios).

Tabla 1. Tipo de documentos publicados

\begin{tabular}{|l|c|c|}
\hline \multicolumn{1}{|c|}{ Tipología de documento } & N & $\%$ \\
\hline Conference Paper & 358 & 47,6 \\
\hline Article & 274 & 36,4 \\
\hline Book Chapter & 41 & 5,5 \\
\hline
\end{tabular}




\begin{tabular}{|l|c|c|}
\hline \multicolumn{1}{|c|}{ Tipología de documento } & N & $\%$ \\
\hline Review & 21 & 2,8 \\
\hline Article in Press & 16 & 2,1 \\
\hline Editorial & 12 & 1,6 \\
\hline Letter & 11 & 1,5 \\
\hline Note & 10 & 1,3 \\
\hline Short Survey & 4 & 0,5 \\
\hline Book & 3 & 0,4 \\
\hline Total & 750 & 99,7 \\
\hline
\end{tabular}

Tras realizar un análisis más pormenorizado sobre la publicación acerca de MOOC según la tipología de documento y el año de publicación se pudo extraer la tendencia mantenida de la presentación de trabajos en formato "conference paper" cuya trayectoria entre 2013 y 2015 ha sido siempre superior el $45 \%$ del total de la productividad sobre el tema por año. En dicho sentido los datos de 2016 parecen indicar que la tendencia se mantendrá o, incluso, aumentará. Por otro lado, el año 2015 ha sido -hasta el momento- el año en que más productividad ha vislumbrado en formato de libro y/o capítulo de libro; confirmando un 8\% de la productividad de 2015 .

Por lo que respecta a la productividad en la categoría artículo, se observa una creciente evolución en términos generales (tabla 2). Aunque el 36,1\% de la productividad anual de 2015 respecto a artículos (y trabajos en prensa) suponga un $3 \%$ menos que el año 2014, cabe resaltar que la productividad en cuanto a número bruto de trabajos es notablemente mayor. En dicho sentido, el panorama de 2016 se configura con un predominio de la productividad respecto a MOOC en el formato de artículos $(63,2 \%)$, con un total de 55 trabajos publicados. En dicho sentido, a 7 meses vista de concluir el año 2016 los datos parecen indicar que las cotas de publicación de artículos serán muy similares a las de 2014 y 2015.

Tabla 2. Tipo de documentos publicados por año

\begin{tabular}{|c|c|c|c|c|c|c|c|c|c|c|}
\hline \multirow{2}{*}{ Tipología de documento } & \multicolumn{2}{|c|}{2012} & \multicolumn{2}{|c|}{2013} & \multicolumn{2}{|c|}{2014} & \multicolumn{2}{|c|}{2015} & \multicolumn{2}{|c|}{2016} \\
\hline & $\mathrm{n}$ & $\%$ & $\mathrm{n}$ & $\%$ & $\mathrm{n}$ & $\%$ & $\mathrm{n}$ & $\%$ & $\mathrm{n}$ & $\%$ \\
\hline Conference Review & o & 0,0 & 1,0 & 1,1 & & 0,0 & & 0,0 & & \\
\hline Coference Paper & 2 & 33,3 & 53,0 & 58,2 & 115,0 & 46,4 & 161,0 & 50,5 & 27,0 & 31,0 \\
\hline Article & 2 & 33,3 & 19,0 & 20,9 & 97,0 & 39,1 & 110,0 & 34,5 & 46,0 & 52,9 \\
\hline Book Chapter & & 0,0 & 1,0 & 1,1 & 15,0 & 6,0 & 25,0 & 7,8 & & 0,0 \\
\hline Review & 1 & 16,7 & 1,0 & 1,1 & 9,0 & 3,6 & 6,0 & 1,9 & 4,0 & 4,6 \\
\hline Article in Press & & 0,0 & & 0,0 & 2,0 & 0,8 & 5,0 & 1,6 & 9,0 & 10,3 \\
\hline Editorial & & 0,0 & 5,0 & 5,5 & 5,0 & 2,0 & 2,0 & 0,6 & & 0,0 \\
\hline
\end{tabular}




\begin{tabular}{|c|c|c|c|c|c|c|c|c|c|c|}
\hline \multirow{2}{*}{ Tipología de documento } & \multicolumn{2}{|c|}{2012} & \multicolumn{2}{|c|}{2013} & \multicolumn{2}{|c|}{2014} & \multicolumn{2}{|c|}{2015} & \multicolumn{2}{|c|}{2016} \\
\hline & $\mathrm{n}$ & $\%$ & $\mathrm{n}$ & $\%$ & $\mathrm{n}$ & $\%$ & $\mathrm{n}$ & $\%$ & $\mathrm{n}$ & $\%$ \\
\hline Letter & & 0,0 & 6,0 & 6,6 & 3,0 & 1,2 & 2,0 & 0,6 & & 0,0 \\
\hline Note & 1 & 16,7 & 5,0 & 5,5 & & 0,0 & 4,0 & 1,3 & & 0,0 \\
\hline Short Survey & & 0,0 & & 0,0 & 2,0 & 0,8 & 2,0 & 0,6 & & 0,0 \\
\hline Book & & 0,0 & & 0,0 & & 0,0 & 2,0 & 0,6 & 1,0 & 1,1 \\
\hline
\end{tabular}

\section{Distribución general de la productividad MOOC por área temática y año}

El análisis de la productividad MOOC por áreas temáticas se llevó a cabo a través de la clasificación de artículos en las áreas establecidas en el Scimago Journal \& Country Rank (SJR). Cabe destacar que SCOPUS permite la multi-clasificación de fuentes y que, por tanto, es probable que algunos de los registros recuperados hayan sido clasificados en varias categorías (una como mínimo). En dicho sentido, el análisis detectó un corpus de clasificación de 1212 trabajos, con lo que 450 trabajos del corpus definitivo $(n=752)$ habían sido clasificados en más de una categoría. En dicho sentido la estadística se calculó en base al corpus de la multi-clasificación con objeto de que los porcentajes se calcularan sobre el 100\% del corpus.

Tal y como se desprendió del análisis global que se ofrece en la tabla 3, dos son los ámbitos o áreas de conocimiento que prestan especial interés a la investigación del fenómeno MOOC. En tal sentido, sobresalen las áreas de Ciencias de la Computación, con un $34,1 \%(n=413)$ de la productividad científica global del fenómeno y, particularmente, las Ciencias Sociales, con 420 trabajos que suponen un $34,7 \%$ de la productividad global. Es destacable también el área de ingeniería, con 118 trabajos y casi un $10 \%$ de la productividad global, y en menor grado, con tasas superior al 3\%, las áreas de Matemáticas y Medicina. Es de especial relevancia indicar que el 100\% de las áreas de clasificación propuestas por SCOPUS han registrado alguna publicación sobre el fenómeno MOOC; cuestión que evidencia el alcance de la temática (tabla 4).

Tabla 3. Productividad por área temática SJR

\begin{tabular}{|l|c|c|}
\hline \multicolumn{1}{|c|}{ Área temática } & $\mathrm{N}$ & $\%$ \\
\hline Agricultural and Biological Sciences & 6 & 0,5 \\
\hline Arts and Humanities & 26 & 2,1 \\
\hline Biochemistry, Genetics and Molecular Biology & 8 & 0,7 \\
\hline Business, Management and Accounting & 29 & 2,4 \\
\hline Chemical Engineering & 1 & 0,1 \\
\hline Chemistry & 5 & 0,4 \\
\hline Computer Science & 413 & 34,1 \\
\hline Decision Sciences & 20 & 1,7 \\
\hline
\end{tabular}




\begin{tabular}{|l|c|c|}
\hline \multicolumn{1}{|c|}{ Área temática } & $\mathrm{N}$ & $\%$ \\
\hline Dentistry & 2 & 0,2 \\
\hline Earth and Planetary Sciences & 3 & 0,2 \\
\hline Economics, Econometrics and Finance & 11 & 0,9 \\
\hline Energy & 2 & 0,2 \\
\hline Engineering & 118 & 9,7 \\
\hline Environmental Science & 6 & 0,5 \\
\hline Health Professions & 10 & 0,8 \\
\hline Immunology and Microbiology & 1 & 0,1 \\
\hline Materials Science & 2 & 0,2 \\
\hline Mathematics & 47 & 3,9 \\
\hline Medicine & 38 & 3,1 \\
\hline Multidisciplinary & 8 & 0,7 \\
\hline Neuroscience & 5 & 0,4 \\
\hline Nursing & 8 & 0,7 \\
\hline Pharmacology, Toxicology and Pharmaceutics & 3 & 0,2 \\
\hline Physics and Astronomy & 3 & 0,2 \\
\hline Psychology & 14 & 1,2 \\
\hline Social Sciences & 420 & 34,7 \\
\hline Veterinary & 3 & 0,2 \\
\hline
\end{tabular}

Del mismo modo, con el objeto de detectar en qué área/s y año/s se había condensado la mayor parte de la productividad científica del fenómeno se realizó un análisis descriptivo en búsqueda de las áreas y años que más habían contribuido a la investigación de los MOOC. En dicho sentido, la tabla 4 aporta los datos de publicación de trabajos por área temática y año, ofreciendo un porcentaje sobre el total de años analizados. Los datos evidencian que la mayor parte de la productividad descrita hasta el momento ha sido publicada en 2015 por el área de Ciencias de la Computación $(15,9 \%)$ y Ciencias Sociales $(15,3 \%)$, respectivamente. En esencia más de $1 / 4$ de la productividad total, es decir, un $31,2 \%(n=379)$ se ha publicado en un año. Cabría también resaltar que casi el 20\% de la productividad global fue desarrollada en 2014 y que, en líneas generales, ambas áreas han incrementado la productividad desde 2012. A fecha actual, parece ser que las Ciencias Sociales aventajan sobre las demás en términos de producción científica sobre el fenómeno MOOC. 
Tabla 4. Productividad por área temática SJR y año con porcentajes sobre la productividad general 2012-2016

\begin{tabular}{|c|c|c|c|c|c|c|c|c|c|c|}
\hline \multirow{2}{*}{ Áreas temáticas } & \multicolumn{2}{|c|}{2012} & \multicolumn{2}{|c|}{2013} & \multicolumn{2}{|c|}{2014} & \multicolumn{2}{|c|}{2015} & \multicolumn{2}{|c|}{2016} \\
\hline & $\mathrm{n}$ & $\%$ & $\mathrm{n}$ & $\%$ & $\mathrm{n}$ & $\%$ & $\mathrm{n}$ & $\%$ & $\mathrm{n}$ & $\%$ \\
\hline $\begin{array}{l}\text { Agricultural and Biological } \\
\text { Sciences }\end{array}$ & & 0,0 & & 0,0 & 6 & 0,5 & & 0,0 & & 0,0 \\
\hline Arts and Humanities & & 0,0 & 3 & 0,2 & 2 & 0,2 & 16 & 1,3 & 5 & 0,4 \\
\hline $\begin{array}{l}\text { Biochemistry, Genetics and } \\
\text { Mol. Biology }\end{array}$ & & 0,0 & 2 & 0,2 & 4 & 0,3 & 1 & 0,1 & 1 & 0,1 \\
\hline $\begin{array}{l}\text { Business, Management and } \\
\text { Accounting }\end{array}$ & 1 & 0,1 & 7 & 0,6 & 14 & 1,2 & 3 & 0,2 & 4 & 0,3 \\
\hline Chemical Engineering & & 0,0 & 1 & 0,1 & & 0,0 & & 0,0 & & 0,0 \\
\hline Chemistry & & 0,0 & 2 & 0,2 & & 0,0 & 2 & 0,2 & 1 & 0,1 \\
\hline Computer Science & 4 & 0,3 & 50 & 4,1 & 119 & 9,8 & 193 & 15,9 & 47 & 3,9 \\
\hline Decision Sciences & & 0,0 & 3 & 0,2 & 9 & 0,7 & 8 & 0,7 & & 0,0 \\
\hline Dentistry & & 0,0 & & 0,0 & & 0,0 & 1 & 0,1 & & 0,0 \\
\hline $\begin{array}{l}\text { Earth and Planetary } \\
\text { Sciences }\end{array}$ & & 0,0 & & 0,0 & 2 & 0,2 & 1 & 0,1 & 1 & 0,1 \\
\hline $\begin{array}{l}\text { Economics, Econometrics } \\
\text { and Finance }\end{array}$ & & 0,0 & 2 & 0,2 & 5 & 0,4 & 1 & 0,1 & 3 & 0,2 \\
\hline Energy & & 0,0 & & 0,0 & & 0,0 & 1 & 0,1 & 1 & 0,1 \\
\hline Engineering & 1 & 0,1 & 7 & 0,6 & 51 & 4,2 & 45 & 3,7 & 14 & 1,2 \\
\hline Environmental Science & & 0,0 & & 0,0 & 3 & 0,2 & 2 & 0,2 & 1 & 0,1 \\
\hline Health Professions & & 0,0 & & 0,0 & 4 & 0,3 & 4 & 0,3 & 2 & 0,2 \\
\hline $\begin{array}{l}\text { Immunology and } \\
\text { Microbiology }\end{array}$ & & 0,0 & & 0,0 & & 0,0 & & 0,0 & 1 & 0,1 \\
\hline Materials Science & & 0,0 & 1 & 0,1 & & 0,0 & & 0,0 & 1 & 0,1 \\
\hline Mathematics & & 0,0 & 6 & 0,5 & 10 & 0,8 & 29 & 2,4 & 2 & 0,2 \\
\hline Medicine & & 0,0 & 3 & 0,2 & 20 & 1,7 & 11 & 0,9 & 4 & 0,3 \\
\hline Multidisciplinary & & 0,0 & 1 & 0,1 & 1 & 0,1 & 5 & 0,4 & 1 & 0,1 \\
\hline Neuroscience & & 0,0 & & 0,0 & 5 & 0,4 & & 0,0 & & 0,0 \\
\hline Nursing & & 0,0 & 1 & 0,1 & 4 & 0,3 & 3 & 0,2 & & 0,0 \\
\hline $\begin{array}{l}\text { Pharmacology, Toxicology } \\
\text { and Phar. }\end{array}$ & & 0,0 & 1 & 0,1 & 1 & 0,1 & & 0,0 & 1 & 0,1 \\
\hline Physics and Astronomy & & 0,0 & 1 & 0,1 & 1 & 0,1 & & 0,0 & 1 & 0,1 \\
\hline Psychology & & 0,0 & & 0,0 & 1 & 0,1 & 9 & 0,7 & 4 & 0,3 \\
\hline Social Sciences & 5 & 0,4 & 50 & 4,1 & 117 & 9,7 & 186 & 15,3 & 62 & 5,1 \\
\hline Veterinary & & 0,0 & 1 & 0,1 & 1 & 0,1 & 1 & 0,1 & & 0,0 \\
\hline
\end{tabular}




\section{Distribución general de la productividad MOOC por países}

El análisis de la productividad MOOC por países reveló datos de especial interés (tabla 5). El 30\% de la productividad actual ha sido elaborada por Estados Unidos (27,4\%) y España (10,6\%). Sin embargo, en un análisis más pormenorizado se ha podido observar como la productividad global de los países miembros de la Unión Europea ha representado el $36,3 \%(n=311)$ de la productividad global; posicionando al Continente Europeo como el principal impulsor de este fenómeno.

Tabla 5. Productividad por países

\begin{tabular}{|l|c|c|}
\hline \multicolumn{1}{|c|}{ País } & N & $\%$ \\
\hline United States & 234 & 27,4 \\
\hline Spain & 91 & 10,6 \\
\hline United Kingdom & 64 & 7,5 \\
\hline China & 58 & 6,8 \\
\hline Australia & 53 & 6,2 \\
\hline Germany & 35 & 4,1 \\
\hline Canada & 27 & 3,2 \\
\hline India & 23 & 2,7 \\
\hline France & 17 & 2,0 \\
\hline
\end{tabular}

*No se incluyen los países con menos de 15 documentos publicados

\section{Distribución general de la productividad MOOC por filiaciones}

Los datos evidenciaron que únicamente tres instituciones contaban con un rango de entre 15 y 20 publicaciones: a) Stanford University $(n=18)$, b) Massachusetts Institute of Technology $(n=15)$ y la Universidad Nacional de Educación a Distancia $(n=14)$. Siguiendo a este grupo encontraríamos aquellas filiaciones que han ha evidenciado una producción de entre 10 y 14 trabajos, a saber: Open University, Pennsylvania State University, Harvard University, Carnegie Mellon University y la Universitat Potsdam. Por lo que respecta al territorio español destacaríamos la Universidad Carlos III de Madrid, Universitat Oberta de Catalunya, Universitat d'Alacant, Universidad Politécnica de Madrid y la Universidad de Salamanca, todas ellas con una producción de entre 5 y 9 trabajos. 


\section{Publicaciones periódicas con más contribuciones}

Tras un análisis de las publicaciones periódicas que más productividad han acumulado sobre la temática (tabla 6) presentamos las 15 fuentes principales. El $46,6 \%(n=7)$ de las mismas corresponden a publicaciones periódicas especializadas en "proceedings" mientras que el resto de publicaciones, el 53,3\% se corresponde a revistas especializadas. Destacar que el $20 \%$ de las publicaciones que a nivel general más han desarrollado el tema son de edición española (Comunicar, Profesorado y RUSC Universities and Knowledge Society Journal).

Tabla 6. Productividad por publicaciones periódicas

\begin{tabular}{|l|c|}
\hline \multicolumn{1}{|c|}{ Publicación } & $\mathrm{n}$ \\
\hline International Review of Research in Open and Distance Learning & 32 \\
\hline $\begin{array}{l}\text { Lecture Notes in Computer Science Including Subseries Lecture Notes in } \\
\text { Artificial Intelligence and Lecture Notes in Bioinformatics }\end{array}$ & 29 \\
\hline ACM International Conference Proceeding Series & 23 \\
\hline L S 2014 Proceedings of the 1st ACM Conference on Learning at Scale & 18 \\
\hline L S 2015 2nd ACM Conference on Learning at Scale & 18 \\
\hline Ceur Workshop Proceedings & 12 \\
\hline Communications in Computer and Information Science & 11 \\
\hline Proceedings Frontiers in Education Conference Fie & 11 \\
\hline $\begin{array}{l}\text { Proceedings of the 2O14 IEEE International Conference on Moocs } \\
\text { Innovation and Technology in Education IEEE Mite 2014 }\end{array}$ & 10 \\
\hline Comunicar & 9 \\
\hline Distance Education & 9 \\
\hline Profesorado & 9 \\
\hline Rusc Universities and Knowledge Society Journal & 9 \\
\hline Mondo Digitale & 8 \\
\hline Computers and Education & 8 \\
\hline
\end{tabular}

\section{Autores, trabajos y citas recibidas}

Con objeto de ofrecer un marco general sobre autores y trabajos más citados respecto a la temática MOOC, se recogieron los cinco autores con mayor impacto (medido en términos de citas). En dicho sentido dos de ellos se posicionaron con más de 150 citas mientras que el resto estuvo comprendido entre 40 y 95 citas (tabla 7). 
Tabla 7. Autores más citados

\begin{tabular}{|l|c|}
\hline \multicolumn{1}{|c|}{ Autor/es } & No citas \\
\hline Liyanagunawardena, T. R., Williams, S. A. & 174 \\
\hline Adams, A. A., & 153 \\
\hline Martin, F. G. & 94 \\
\hline Jordan, K. & 58 \\
\hline Guo, P. J., Kim, J., Rubin, R. & 41 \\
\hline
\end{tabular}

Por último, se rescataron aquellos trabajos de revistas (excluyendo la categoría "proceedings") que mayor número de citas habían recibido, recuperando al igual que anteriormente los cinco trabajos más citados hasta el momento según la base de datos SCOPUS (tabla 8).

Tabla 8. Artículos más citados

\begin{tabular}{|c|c|c|c|c|}
\hline Título artículo & Autores & Revista & Año & Citas \\
\hline $\begin{array}{l}\text { MOOCs: A systematic } \\
\text { study of the published } \\
\text { literature } 2008-2012\end{array}$ & $\begin{array}{l}\text { Liyanagunawardena, } \\
\text { T.R., Adams, A.A., } \\
\text { Williams, S.A. }\end{array}$ & $\begin{array}{l}\text { International Review of } \\
\text { Research in Open and } \\
\text { Distance Learning }\end{array}$ & 2013 & 153 \\
\hline $\begin{array}{l}\text { Education will massive } \\
\text { open online courses } \\
\text { change how we teach }\end{array}$ & Martin, F.G. & $\begin{array}{l}\text { Communications of the } \\
A C M\end{array}$ & 2012 & 94 \\
\hline $\begin{array}{l}\text { Initial trends in } \\
\text { enrolment and } \\
\text { completion of massive } \\
\text { open online courses }\end{array}$ & Jordan, K. & $\begin{array}{l}\text { International Review of } \\
\text { Research in Open and } \\
\text { Distance Learning }\end{array}$ & 2014 & 58 \\
\hline $\begin{array}{l}\text { Changing "Course": } \\
\text { Reconceptualizing } \\
\text { Educational Variables } \\
\text { for Massive Open } \\
\text { Online Courses }\end{array}$ & $\begin{array}{l}\text { DeBoer, J., Ho, A.D., } \\
\text { Stump, G.S., Breslow, L. }\end{array}$ & Educational Researcher & 2014 & 38 \\
\hline $\begin{array}{l}\text { MOOCs: So many } \\
\text { learners, so much } \\
\text { potential. }\end{array}$ & $\begin{array}{l}\text { Kay, J., Reimann, } \\
\text { P., Diebold, E., } \\
\text { Kummerfeld, B. }\end{array}$ & $\begin{array}{l}\text { IEEE Intelligent } \\
\text { Systems }\end{array}$ & 2013 & 37 \\
\hline
\end{tabular}

\section{Indicadores de co-citación}

A través del software VOSwiewer se analizaron las principales relaciones existentes entre los documentos del corpus de trabajos definitivos extraídos a través de SCOPUS. El análisis de co-citación evidenció la relación entre las publicaciones más relevantes, no en términos de citas, sino en base a la citación conjunta por los 
artículos recuperados en la muestra del estudio, evidenciado así su relevancia en la temática MOOC (figura 1).

Figura 1. Publicaciones con más de 10 co-citaciones

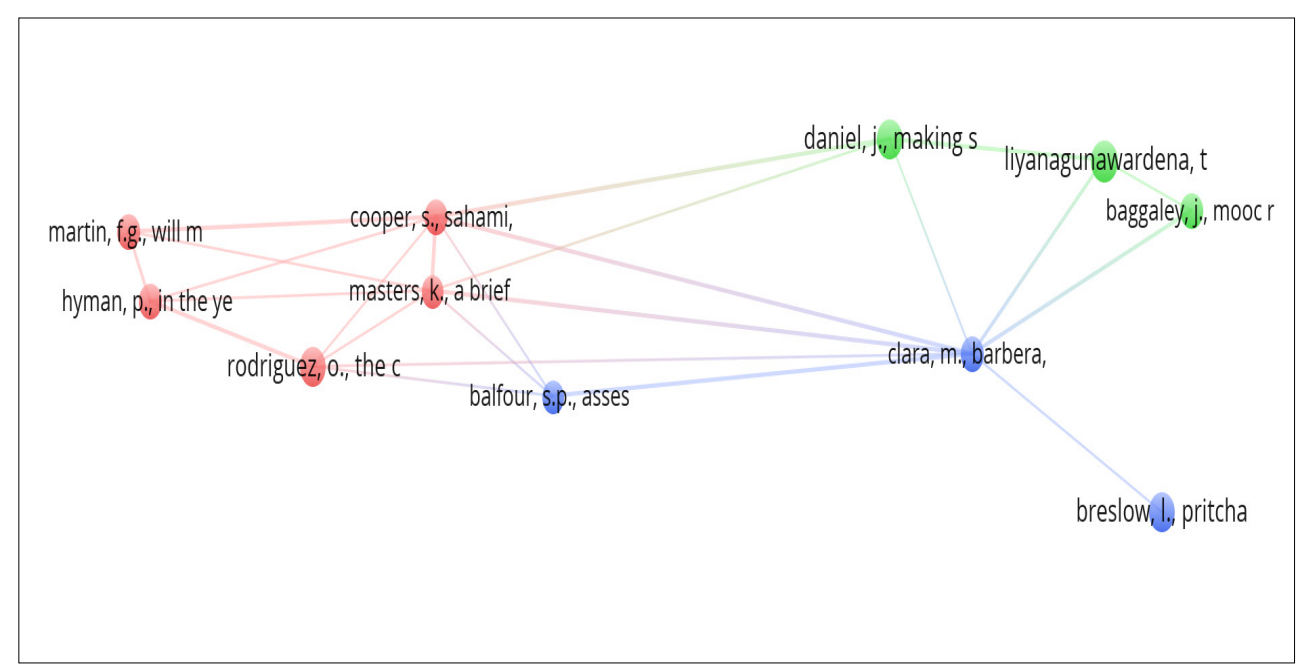

En dicho sentido se extrae que, en base a la co-citación, las publicaciones más relevantes sobre MOOC según los datos extraídos de SCOPUS son:

- Clarà, M., y Barberà, E. (2013). Learning online: Massive open online courses (MOOCs), connectivism, and cultural psychology. Distance Education, 34(1), 129-136.

- Cooper, S., y Sahami, M. (2013). Education reflections on stanford's MOOCs. Communications of the ACM, 56(2), 28-30.

- Masters, K. (2011). A Brief Guide To Understanding MOOCs. The Internet Journal of Medical Education, 1(2).

- Daniel, J. (2012). Making Sense of MOOCs: Musings in a Maze of Myth, Paradox and Possibility. Journal of Interactive Media in Education, 2012(3), Art. 18.

- Liyanagunawardena, T. R., Adams, A. A., y Williams, S. A. (2013). MOOCs: A systematic study of the published literature 2008-2012. International Review of Research in Open and Distance Learning, 14(3), 202-227. 


\section{CONCLUSIONES}

El presente trabajo ha analizado la productividad científica alrededor del fenómeno MOOC desde 2012 hasta 2016 a partir de los datos obtenidos de la base de datos SCOPUS. El trabajo ha permitido, entre otros, obtener una visión global de la investigación sobre MOOC y su evolución en los últimos 5 años en función de las áreas temáticas, países y tipos de documento. Del mismo modo, el trabajo amplía considerablemente la visión ofrecida por trabajos recientes y similares (Zancanaro y Domingues, 2017; López-Meneses, Vázquez-Cano y Román, 2015; Aguaded, Vázquez-Cano y López-Meneses, 2016), pues ofrece una cobertura mayor (número de años y documentos) y específica de la base de datos SCOPUS, con mayor cobertura en la temática analizada que la base de datos WOS.

Los resultados de la presente investigación corroboran los resultados de actuales trabajos similares más centrados en el panorama tanto español (Aguaded-Gómez, Vázquez-Cano y López-Meneses, 2016; López-Meneses, Vázquez-Cano y Román, 2015) como iberoamericano (Zancanaro y Domingues, 2017). Al mismo tiempo, complementa estudios anteriores (Daradoumis, Bassi, Xhafa y Caballé, 2013; Liyanagunawardena, Adams y Williams, 2013).

En primer lugar, se ha evidenciado una productividad científica destacada, que con una evaluación constante que prevé que el 2016 se posicione, si cabe, al mismo nivel que los años 2014 y 2015. En dicho sentido, este estudio coincide con otros trabajos (e.j: Zancarano y Domingues, 2017) en resaltar que el fenómeno MOOC se consolida como área de investigación y tiene su punto de partida en 2012. Esta cuestión pone de manifiesto la vigencia de la temática MOOC y la confrontación con los investigadores que describen la ya era post-MOOC. Con todo el análisis realizado pone de manifiesto que la actualidad de la temática se manifiesta a través de dos fuentes básicas de difusión: a) el artículo científico y b) los papers de comunicaciones indexadas. Del mismo modo, el escenario en 2016 pareciera ser que acogerá en mayor medida el artículo científico como medio de difusión del conocimiento respecto a MOOC, sí y solo sí los datos de productividad evidenciados por SCOPUS mantienen la tendencia actual.

Asimismo, se ha evidenciado que los campos de las Ciencias Sociales -en particular- y las Ciencias de la Computación son aquellos más activos en la investigación y dinamización del conocimiento respecto a los MOOC, habiendo producido un $31,2 \%$ de la productividad científica total del fenómeno tan solo en el año 2015, más un 20\% acontecida en 2014. En dicho sentido el fenómeno MOOC se ha considerado global, tanto es que el análisis realizado vislumbra una cobertura del $100 \%$ de todas las áreas temáticas de la clasificación empleada por el SJR, siendo dos países los que abanderan las mayores tasas de productividad: Estados Unidos, con un 27,4\% del total y España, con un 10,6\%. En la misma línea, los datos han evidenciado la excelente contribución española al fenómeno. No solo por ser el segundo país en productividad, también por contar con diversas filiaciones de 
entre las más productivas en este ámbito, y por albergar el 20\% de las publicaciones periódicas que más han investigado al respecto.

En última instancia, los análisis finales han ofrecido un marco de autores y trabajos más citados mucho más rico que trabajos anteriormente publicados sobre la temática. El análisis de co-citación ha evidenciado aquellas publicaciones más relevantes en la temática a partir del análisis de citación conjunta del corpus analizado. Por lo tanto, el presente estudio se configura como una herramienta de reflexión en torno a la configuración de una nueva modalidad de aprendizaje que, sin duda, ha suscitado el movimiento de la comunidad científica. Por otro lado, fruto de las limitaciones que el presente estudio puede tener (falta de actualización de datos por parte de SCOPUS, necesidad de comparación con otras bases de datos como WOS) se configura como una línea de trabajo emergente que puede ser retomada, como bien decimos, empleando otras técnicas de análisis y bases de datos de consulta. En cualquier caso, los estudios bibliométricos son de especial interés para la comunidad académica; no solo porque fomentan la promoción de la investigación sobre los MOOC sino también porque ofrecen un panorama general del crecimiento de la ciencia en general y de un ámbito concreto, en particular.

\section{REFERENCIAS BIBLIOGRÁFICAS}

Aguaded-Gómez, I., Vázquez-Cano, E., y López-Meneses, E. (2016). El impacto bibliométrico del movimiento MOOC en la Comunidad Científica Española. Educación XX1, 19(2), 77-104. doi: 10.5944/educXX1. 13217

Alcaide, G. G., Far, A. C., e Iglesias, E. B. (2014). Núcleos y ámbitos de investigación sobre adicciones en España a través del análisis de los enlaces bibliográficos en la Web of Science (2000-2013). Adicciones: Revista de socidrogalcohol, 26(2), 168183.

Anderson, T. (2013). Promise and/or Peril: $M O O C$ and Open and Distance Education. Commonwealth of Learning. Recuperado de http://www. col.org/SiteCollectionDocuments MOOCPromisePeril Anderson.pdf

Ariza, T., Granados, M. R., Ramiro, M. T., y Gómez-García, A. (2011). Una década de la Revista Española de Orientación y Psicopedagogía: un análisis bibliométrico de su evolución. Revista Española de Orientación y Psicopedagogía, 22, 38-57.

Castaño-Muñoz, J., Duart, J., y Teresa, S. (2015). Determinants of Internet use for interactive learning: an exploratory study. Journal of New Approaches in Educational Research, 4(1), 25-34.

Christensen, G., Steinmetz, A., Alcorn, B., Bennett A., Woods, D., y Emanuel, E. J. (2013). The MOOC phenomenon: Who takes massive open online courses and why? Working Paper. University of Pennsylvania. Recuperado de http://papers.ssrn.com/sol3/papers. cfm?abstract id=2350964

Colorado, A., Marín-Díaz, V., y Zavala, Z. (2016). Impacto del grado de apropiación tecnológica en los estudiantes de la Universidad Veracruzana International Journal of Educational Research and Innovation (IJERI), 5, 124-137.

Conole, G. (2013). Designing for learning in an open world. New York: Springer. 
Cormier, D., y Siemens, G. (2010). Through the open door: open courses as research, learning, and engagement. EDUCAUSE Review, 45(4), 30-39.

Daniel J. (2012). Making Sense of MOOCs: Musings in a Maze of Myth, Paradox and Possibility.JournalofInteractive Mediain Education, 3. Recuperado de http://iime. open.ac.uk/articles/10.5334/2012-18/

Daniel, J., Vázquez-Cano, E., y Gisbert, M. (2015). The future of MOOCs: Adaptative Learning or Business Model? RUSC. Universities and Knowledge Society Journal, 12(1), 64-73.

Daradoumis, T., Bassi, R., Xhafa, F., y Caballé, S. (2013). A review on massive e-learning (MOOC) design, delivery and assessment. Paper presented at the Proceedings of 8th International Conference on P2P, Parallel, Grid, Cloud and Internet Computing, 3PGCIC 2013, 208-213. doi: 10.1109/3PGCIC.2013.37

Davis, J. C., y Gonzalez, J. G. (2003). Scholarly Journal Articles about the Asian Tiger Economies: Authors, Journals, and Research Fields, 1986-2001. AsianPacific Economic Literature, 17, 51-61

DeBoer, J., Ho, A., Stump, G., y Breslow, L. (2014). Changing "course:" reconceptualizing educational variables for massive open online courses. Educational Researcher, 43(2), 74-84.

Dillenbourg, P., Fox, A., Kirchner, C., Mitchell, J., y Wirsing, M. (Eds.). (2014). Massive open online courses: current state and perspectives. Dagstuhl Manifestos. Schloss Dagstuhl - Leibniz Zentrum für Informatik.

Downes, S. (2012). The Rise of MOOC. Recuperado de http://www.downes.ca/ post/57911

Downes, S. (2013). The Quality of Massive Open Online Courses. Recuperado de http://mooc.efquel.org/week-2-thequality-of-massive-open-online-coursesby-stephen-downes
Eaton, J. (2012). MOOC and Accreditation: Focus on the Quality of "Direct-toStudents". Education Council for Higher Education Accreditation, 9(1). Recuperado de www.chea.org/ia IA 2012.10.31.htm

Estévez, J., y García, A. (2015). Las redes sociales para la mejora de la capacidad de emprender y de autoempleo International Journal of Educational Research and Innovation (IJERI), 4, 101-110.

Fernández Cano, A. (2011). Producción educativa española en el Social Sciences Citation Index (1998-2009). Revista Española de Pedagogía, 69(250), 427443.

Fernández Cano, A., y Bueno Sánchez, A. (1998). Síntesis de estudios bibliométricos españoles en educación. Una dimensión evaluativa. Revista española de documentación científica, 21(3), 269-285.

Gómez-García, A., Ramiro, M. T., Ariza, T., y Granados, M. R. (2012). Estudio bibliométrico de Educación XX1. Educación XX1, 15, 17-41.

Gisbert Cervera, M., y Lázaro Cantabrana, J. (2015). Professional development in teacher digital competence and improving school quality from the teachers' perspective: a case study. Journal of New Approaches in Educational Research, 4(1), 115-122.

Guàrdia, L., Maina, M., y Sangrà, A. (2013). MOOC Design Principles. A Pedagogical Approach from the Learner's Perspective. eLearning Papers, 33. Recuperado de http://www.openeducationeuropa.eu en/article/MOOC-Design-Principles. A-Pedagogical-Approach-from-theLearner\%E2\%80\%99s-Perspective

Hoxby, C. M. (2014). The economics of online postsecondary education: MOOCs, nonselective education, and highly selective education. NBER Working Paper 19816. Recuperado de http://www. nber.org/papers/w19816 
Knoke, D., y Yang, S. (Eds.). (2008). Social network analysis, 154 . Sage.

Liyanagunawardena, T. R., Adams, A. A., y Williams, S. A. (2013). MOOCs: A systematic study of the published literature 2008-2012. International Review of Research in Open and Distance Learning, 14(3), 202-227.

Little, G. (2013). Massively Open? The Journal of Academic Librarianship, 39(3), 308-309.

López-Meneses, E., Vázquez-Cano, E., y Román, P. (2015). Analysis and implications of the impact of MOOC movement in the scientific community: JCR and Scopus (2010-13). Comunicar, 22(44), 73-80. doi: 10.3916/C44-201508.

López Meneses, E. Vázquez-Cano, E., y Sarasola J. L. (2015). Estudio Bibliométrico Pixel-Bit. Revista de Medios y Educación (2000-2013). PixelBit. Revista de Medios y Educación, 46, 65-85.

Marín Díaz, V., y Sampedro Requena, B. E. (2016). Web 2.0 for the invigoration and participation of families and communities. Journal of New Approaches in Educational Research, 5(1), 38-43. doi: 10.7821/naer.2016.1.137

Mengual-Andrés, S., Roig-Vila, R., y Lloret Catalá, C. (2015). Validación del cuestionario de evaluación de la calidad de cursos virtuales adaptado a MOOC. RIED. Revista Iberoamericana De Educación a Distancia, 18(2), 145-169.

Pappano, L. (2012). Year of the MOOC. New York Times. Recuperado de http://www.nytimes.com/2012/11/04/ education/edlife/massive-open-onlinecourses-are-multiplving-at-a-rapid-pace html?pagewanted=all\& $\mathrm{r}=$ oPappano

Peritz, B. C., y Bar-Ilan, J. (2002). The sources used by bibliometrics scientometrics as reflected in references. Scientometrics, 54(2), 269-284.
Radford, A. W., Robles, J., Cataylo, S., Horn, L., Thornton, J., y Whitfield, K. (2014). The employer potential of MOOCs: a survey of human resource professionals' thinking on MOOCs. RTI International. Recuperado de http://www.rti.org/pubs/ duke handbook-final-03252014.pdf

Ramírez-Fernández, M. B. (2015). Propuesta de certificación de calidad de la oferta española educativa de cursos MOOC. International Journal of Educational Research and Innovation (IJERI), 3, 121133.

Roig Vila, R., Mengual Andrés, S., y Suárez Guerrero, C. (2014). Evaluación de la calidad pedagógica de los MOOC Profesorado. Revista de Currículum y Formación de Profesorado, 18(1), 27-41.

Roig-Vila, R., Mondéjar, L., y Lorenzo-Lledó, G. (2016). Redes sociales científicas. La Web social al servicio de la investigación. International Journal of Educational Research and Innovation (IJERI), 5, 171183.

Sangrà, A. (2013). Retos de los MOOCs. Recuperado de http://www.youtube. com/watch?v=EVbjU8yi7Ow

Siemens, G. (2012). MOOCs for the win! ElearnSpace. Recuperado de http:/ www.elearnspace.org/blog/2012/03/05/ moocs for-the-win/

Yuan, L., y Powell, S. (2013). MOOCs and Open Education: Implications for Higher Education. Recuperado de http:// publications.cetis.ac.uk/2013/667

UNESCO (2013). Policy guidelines for mobile learning. Paris: United Nations Educational, Scientific and Cultural Organization.

Vázquez-Cano, E. (2013). The Videoarticle: New Reporting Format in Scientific Journals and its Integration in MOOCs. Comunicar, 41, 83-91. doi: http://dx.doi. org/10.3916/C41-2013-08

Vázquez-Cano, E., López-Meneses, E., y Sarasola, J. L. (2013). La expansión del 
conocimiento en abierto: Los MOOCs. Barcelona: Octaedro.

Vázquez-Cano, E., y Sevillano, M. L. (2015). Analysis of risks in a Learning Management System: A case study in the Spanish National University of Distance Education (UNED). New Approaches in Educational Research, 4(1), 62-68.

Vázquez-Cano, E., López Meneses, E., y Cobos Sánchiz, D. (2015). Estudio Bibliométrico de Profesorado. Revista de Currículum y formación del profesorado (1997-2013). Revista de Currículum y formación del profesorado, 18(3), 191-212.

Vázquez-Cano, E., López, E., y Barroso Osuna, J. (2015). El futuro de los MOOC: Retos de la formación on-line, masiva y abierta. Madrid: Síntesis.
Zancanaro, A., Todesco, J. L., y Ramos, F. (2015). A bibliometric mapping of open educational resources. The International Review of Research in Open and Distributed Learning, 16(1).

Zancanaro, A., y Domingues, M. (2017). Analysis of the scientific literature on Massive Open Online Courses (MOOCs)/ Análisis de la literatura científica sobre los cursos en línea abiertos y masivos (MOOC). RIED. Revista Iberoamericana de Educación a Distancia, 20(1). doi: 10.5944/ried.20.1.15910

Zapata, M. (2013). MOOC, una visión crítica. El valor no está en el ejemplar. Recuperado de http://eprints.rclis. org/18452

\section{PERFIL ACADÉMICO Y PROFESIONAL DE LOS AUTORES}

Santiago Mengual-Andrés. Doctor y licenciado en Psicopedagogía. Profesor contratado doctor del Dpto. de Educación Comparada e Historia de la Educación de la Facultad de Filosofía y Ciencias de la Educación de la Universidad de Valencia. Ha sido secretario académico de la delegación de la Comunidad Valenciana de la Asociación Interuniversitaria de Investigación Pedagógica (AIDIPE). Es editor ejecutivo del Journal of New Approches in Educational Research. Su investigación se centra en el análisis de políticas nacionales e internacionales de inclusión TIC, competencia digital y nuevos escenarios de educación con tecnología. Ha participado y participa como docente en diversos másteres y programas de posgrado de ámbito nacional e internacional.

E-mail: santiago.mengual@ua.es

\section{DIRECCIÓN DEL AUTOR}

Dpto. de Educación Comparada e Historia de la Educación

Facultad de Filosofía y Ciencias de la Educación

Universidad de Valencia

Av. Blasco Ibáñez, 30 (46010)

Valencia (España)

Esteban Vázquez-Canon. Profesor de la Facultad de Educación en el Departamento de Didáctica, Organización Escolar y Didácticas Especiales de la 
Universidad Nacional de Educación a Distancia (UNED) con acreditación de profesor titular de universidad. Su trayectoria profesional ha estado siempre dedicada a la educación como profesor de enseñanza secundaria e inspector de educación. Es miembro de diferentes proyectos de investigación nacionales e internacionales que tienen como temática principal el aprendizaje ubicuo y móvil con dispositivos digitales. Sus líneas de investigación prioritarias son la organización y supervisión escolar, la didáctica con TIC, el aprendizaje móvil-ubicuo, el lenguaje digital y los cursos online masivos en abierto (MOOC); temáticas sobre las que ha publicado más de 100 contribuciones científicas.

E-mail: evazquez@edu.uned.es

\section{DIRECCIÓN DEL AUTOR}

Departamento de Didáctica, Organización Escolar y Didácticas Especiales.

Universidad Nacional de Educación a Distancia

C/ Juan del Rosal, 14 (28040)

Madrid (España)

Eloy López Meneses. Profesor titular de universidad en el Departamento de Educación y Psicología Social de la Universidad Pablo de Olavide (Sevilla, España) con más de quince años en la docencia universitaria sobre TIC. Doctor en Ciencias de la Educación y premio extraordinario de tesis doctoral por la Universidad de Sevilla. Segundo premio nacional en los estudios de Ciencias de la Educación. Miembro e investigador del Grupo de Investigación Didáctica (GID), desde el año 1999 (HUM0390). Desde enero de 2005, miembro del grupo de investigación: Nodo Educativo www.nodoeducativo.org. Investigador en más de veintidós proyectos competitivos. Entre sus libros se encuentran: "Evaluación de materiales multimedia en red en el Espacio Europeo de Educación Superior" (2009), Barcelona: DaVinci; "La expansión del conocimiento en abierto: los MOOC". (2013). Barcelona: Editorial Octaedro. E-mail: elopmen@upo.es

\section{DIRECCIÓN DEL AUTOR}

Dpto. de Educación y Psicología Social. Área de Didáctica y Organización Escolar Universidad Pablo de Olavide Ctra. de Utrera, km. 1 (41013)

Sevilla (España)

Fecha de recepción del artículo: 29/05/2016

Fecha de aceptación del artículo: 19/09 /2016 


\section{Como citar este artículo:}

Mengual-Andrés, S., Vázquez-Cano, E., y López Meneses, E. (2017). La productividad científica sobre MOOC: aproximación bibliométrica 2012-2016 a través de SCOPUS. RIED. Revista Iberoamericana de Educación a Distancia, 2O(1), pp. 39-58. doi: http://dx.doi.org/10.5944/ried.20.1.16662 